\title{
Produção endodôntica no atendimento público odontológico do Centro-Oeste do Brasil
}

\author{
Thiago Machado Pereira*; Alvaro Henrique Borges*; Laura Maria de Amorim Santana Costa*; \\ Durvalino Oliveira*; Luiz Evaristo Ricci Volpato*
}

* Departamento de Ciências Orais, Universidade de Cuiabá

Recebido em 31/10/2017. Aprovado em 21/03/2018.

\begin{abstract}
RESUMO
Pouco se sabe sobre o atendimento público odontológico no Centro-Oeste do Brasil, ainda menos sobre sua produção endodôntica. Esta produção é realizada principalmente nos Centros de Especialidades Odontológicas (CEO), referência pública para tratamento endodôntico, acessível na base de dados DATASUS. Foi objetivo deste estudo explorar a produção endodôntica nas capitais dos estados da Região Centro-Oeste e do Distrito Federal do Brasil. Este estudo retrospectivo transversal analisou a produção endodôntica destes municípios de janeiro de 2011 a dezembro de 2016. Os dados foram obtidos consultando as bases do Ministério da Saúde (DATASUS) e do Instituto Brasileiro de Geografia e Estatística (IBGE). Os valores foram comparados por análise de variância e os testes de Kolmogorov-Smirnov e Levene seguidos pelo teste post hoc de Tukey. O nível de significância foi definido em $\mathrm{p}<0,05$. Dentre as cidades analisadas, Campo Grande teve a maior produção endodôntica por habitante, seguida de Cuiabá e Goiânia com produções similares, e Brasília. Os dentes com três ou mais canais radiculares apresentaram maior incidência de tratamento endodôntico comparados a dentes com um ou dois canais radiculares. A produção endodôntica dos CEO das capitais dos estados da Região Centro-Oeste e do Distrito Federal do Brasil foi proporcionalmente maior em Campo Grande e menor em Brasília. O tratamento endodôntico foi realizado preferencialmente nos dentes com três ou mais canais radiculares. As instituições de ensino devem estar atentas às mudanças para que seus egressos sejam formados em consonância com o perfil epidemiológico da população bem como com as políticas públicas de saúde.

Descritores: Endodontia. Odontologia. Sistema Único de Saúde.
\end{abstract}




\section{INTRODUÇÃO}

Nas últimas décadas, a assistência pública odontológica no Brasil passou por transições em seu perfil ${ }^{1-3}$. Anteriormente conhecida como mutiladora, obteve com o advento da Política Nacional de Saúde Bucal, evolução considerável na qualidade de sua assistência ${ }^{2,3}$. Uma vez que o perfil mutilador da atenção básica se caracteriza pela concentração nas extrações dentárias, a elevação da produção de tratamentos conservadores alternativos, como os tratamentos endodônticos, pode representar mudança de paradigma $^{4-6}$.

A academia deve estar atenta às possíveis modificações no perfil da assistência odontológica oferecida pelo Sistema Único de Saúde (SUS) e se reorientar por essas mudanças, visto que a assistência é um ordenador do perfil de recursos humanos a serem inseridos no mercado de trabalho ${ }^{7}$. Assim, não apenas é importante que haja uma imersão do acadêmico no SUS por meio de diferentes estratégias, como por exemplo os estágios extramuros, como também que os mesmos sejam preparados para analisar criticamente a realidade em que estão inseridos ${ }^{7,8}$.

A manutenção dos dentes permite a continuidade na realização de sua função, afetando positivamente a saúde do indivíduo 9 . Por outro lado, como o tratamento endodôntico é muito mais complexo que a extração de dentes danificados, sua implementação em larga escala na saúde pública exige não apenas uma mudança de filosofia, mas também de investimento ${ }^{5,10}$. Portanto, a alta prevalência de tratamentos endodônticos pode refletir esse sinal de mudança ${ }^{4}$.

Pouco se sabe sobre o atendimento público odontológico no Centro-Oeste brasileiro, ainda menos sobre sua produção endodôntica ${ }^{6,11,12}$. Esta produção é realizada principalmente nos
Centros de Especialidades Odontológicas (CEO), referência pública para tratamento endodôntico, acessível pela base de dados DATASUS $^{13}$. Esta apresenta fácil acesso e eficiência na obtenção de dados e informações em saúde pública, estando disponível para qualquer gerente, profissional ou pesquisador ${ }^{13}$. O objetivo deste estudo foi explorar a produção endodôntica nas capitais dos estados da Região Centro-Oeste do Brasil.

\section{METODOLOGIA}

Este estudo foi aprovado pelo comitê de ética em pesquisa da Universidade de Cuiabá CAAE: 68813517.5.0000.5165. Caracteriza-se como transversal retrospectivo e analisou a produção endodôntica das capitais dos estados do Centro-Oeste e do Distrito Federal no período compreendido entre janeiro de 2011 e dezembro de 2016.

Os dados foram obtidos por meio de consultas nas bases de dados do Ministério da Saúde $\left(\right.$ DATASUS) ${ }^{14}$. A pesquisa seguiu os indicadores: informações sobre saúde (TABNET); produção ambulatorial (SIASUS); local de serviço - a partir de 2008; e escolha do estado. Em seguida, as variáveis selecionadas foram: município de origem (Brasília - Distrito Federal, Campo Grande - Mato Grosso do Sul, Cuiabá - Mato Grosso, Goiânia - Goiás), período (janeiro de 2011 a dezembro de 2016) e tipo de procedimento (tratamento endodôntico em dentes permanentes com um canal radicular, dois canais radiculares e com três ou mais canais radiculares).

A tabulação foi feita por meio do Programa Tab para Windows - TABWIN (Ministério da Saúde, Brasília, Brasil) e transcritas em tabelas e gráficos usando o Microsoft Excel 15.0 - Office 2013 (Microsoft Corp., Redmond, EUA). 
Os dados da população foram extraídos do Instituto Brasileiro de Geografia e Estatística (IBGE) no site www.ibge.gov.br.

Os valores foram comparados por análise de variância e testes de Kolmogorov-Smirnov e Levene, seguidos pelo teste post hoc de Tukey. O nível de significância foi definido em $\mathrm{p}<0,05$. Todas as análises estatísticas foram realizadas usando SPSS versão 18.0 para Windows (SPSS Inc, Chicago, EUA).

\section{RESULTADOS}

Campo Grande apresentou maior produção endodôntica por habitante, seguida de Cuiabá e Goiânia com produções similares, e Brasília. A produção endodôntica das capitais dos estados do Centro-Oeste do Brasil de 2011 a 2016 é apresentada na tabela 1. A tabela 2 descreve a população das capitais dos estados do CentroOeste do Brasil, a produção total de tratamentos endodônticos realizados de 2011 a 2016 e o índice de tratamento e população. A figura 1 apresenta a produção endodôntica nos municípios de acordo com os diferentes grupos dentários.

Tabela 1. Produção endodôntica no atendimento público odontológico nas capitais estaduais do Centro-Oeste de 2011 a 2016

\begin{tabular}{|c|c|c|c|c|c|c|c|}
\hline Procedimento/Ano & 2011 & 2012 & 2013 & 2014 & 2015 & 2016 & Total \\
\hline \multicolumn{8}{|l|}{ Brasília, DF } \\
\hline $\begin{array}{l}\text { Dentes permanentes com um } \\
\text { canal radicular }\end{array}$ & 860 & 1.172 & 1.090 & 1.064 & 1.133 & 977 & 6.296 \\
\hline $\begin{array}{l}\text { Dentes permanentes com dois } \\
\text { canais radiculares }\end{array}$ & 497 & 772 & 699 & 756 & 600 & 814 & 4.138 \\
\hline $\begin{array}{l}\text { Dentes permanentes com três } \\
\text { ou mais canais radiculares }\end{array}$ & 1.113 & 1.572 & 1.433 & 1.378 & 1.294 & 1.294 & 8.084 \\
\hline \multicolumn{8}{|l|}{ Campo Grande, MS } \\
\hline $\begin{array}{l}\text { Dentes permanentes com um } \\
\text { canal radicular }\end{array}$ & 2.593 & 2.241 & 2.657 & 2.794 & 2.743 & 2.558 & 15.586 \\
\hline $\begin{array}{l}\text { Dentes permanentes com dois } \\
\text { canais radiculares }\end{array}$ & 2.187 & 1.857 & 2.372 & 2.820 & 2.781 & 2.514 & 14.531 \\
\hline $\begin{array}{l}\text { Dentes permanentes com três } \\
\text { ou mais canais radiculares }\end{array}$ & 3.815 & 3.513 & 4.049 & 4.515 & 4.644 & 4.013 & 24.549 \\
\hline \multicolumn{8}{|l|}{ Cuiabá, MT } \\
\hline $\begin{array}{l}\text { Dentes permanentes com um } \\
\text { canal radicular }\end{array}$ & 599 & 816 & 1108 & 1027 & 904 & 906 & 5.360 \\
\hline $\begin{array}{l}\text { Dentes permanentes com dois } \\
\text { canais radiculares }\end{array}$ & 474 & 717 & 748 & 789 & 709 & 656 & 4.093 \\
\hline $\begin{array}{l}\text { Dentes permanentes com três } \\
\text { ou mais canais radiculares }\end{array}$ & 596 & 992 & 1405 & 1322 & 1606 & 1273 & 7.194 \\
\hline \multicolumn{8}{|l|}{ Goiânia, GO } \\
\hline $\begin{array}{l}\text { Dentes permanentes com um } \\
\text { canal radicular }\end{array}$ & 2.455 & 2.635 & 2.355 & 2.121 & 1.070 & 818 & 11.455 \\
\hline $\begin{array}{l}\text { Dentes permanentes com dois } \\
\text { canais radiculares }\end{array}$ & 2.286 & 2.614 & 2.086 & 2.608 & 1.123 & 715 & 11.432 \\
\hline $\begin{array}{l}\text { Dentes permanentes com três } \\
\text { ou mais canais radiculares }\end{array}$ & 2.782 & 2.961 & 2.593 & 3.459 & 1.612 & 1.040 & 14.447 \\
\hline
\end{tabular}


Tabela 2. População de capitais estaduais do Centro-Oeste do Brasil, produção total de tratamentos endodônticos realizados entre 2011 e 2016 e a média de tratamento por população

\begin{tabular}{cccc}
\hline Município & População* & $\begin{array}{c}\text { Quantidade de } \\
\text { tratamentos } \\
\text { endodônticos }\end{array}$ & $\begin{array}{c}\text { Número de } \\
\text { tratamentos/ } \\
\text { População }\end{array}$ \\
\hline Brasília & 2.977 .216 & 18.518 & $0,006^{\mathrm{a} * *}$ \\
Campo Grande & 863.982 & 54.666 & $0,063^{\mathrm{c}}$ \\
Cuiabá & 585.367 & 16.647 & $0,028^{\mathrm{b}}$ \\
Goiânia & 1.448 .639 & 37.334 & $0,026^{\mathrm{b}}$ \\
\hline
\end{tabular}

* Os dados da população foram extraídos do IBGE (Instituto Brasileiro de Geografia e Estatística) no site www.ibge.gov.br (acessado em 24/05/2017).

** Letras diferentes representam diferenças estatísticas.

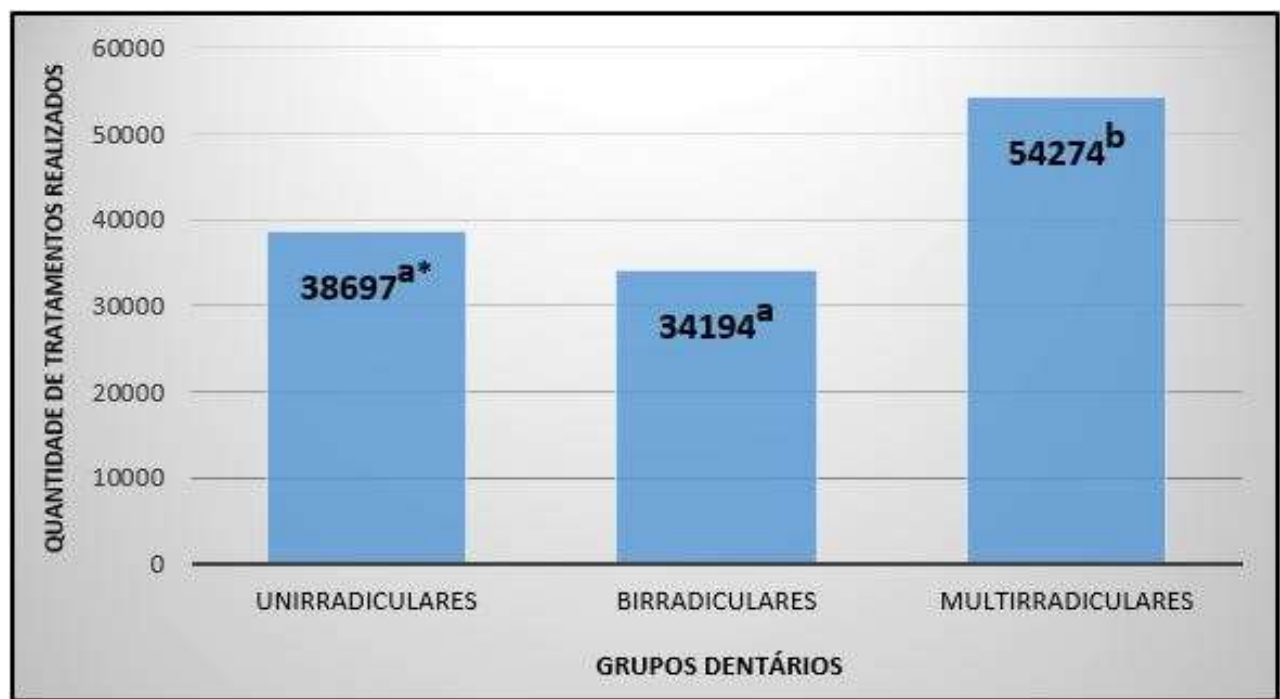

Figura 1. Produção endodôntica em diferentes grupos dentários na região centro-oeste brasileira de 2011 a 2016

* Letras diferentes representam diferenças estatísticas.

\section{DISCUSSÃO}

Este estudo demonstra que o atendimento público odontológico brasileiro não pode ser definido de forma estritamente mutiladora. A quantidade de tratamentos endodônticos realizados nas quatro cidades analisadas mostra claramente que há preocupação e investimento na mudança deste paradigma. No entanto, estes resultados devem ser considerados com cautela, uma vez que não estão embasados em estudos epidemiológicos que analisem as necessidades de tratamento da população e que a assistência odontológica nas capitais pode não refletir a realidade dos demais municípios de cada estado $^{15,16}$.

Os resultados também demonstram que a produção endodôntica é diferente nas cidades consultadas. Como a população das capitais é bastante diversa, a relação do tratamento endodôntico por população foi realizada para 
permitir a comparação entre as cidades. Ao fazêlo, pode-se ver que Campo Grande tem a maior produção endodôntica por habitante, seguida de Cuiabá e Goiânia com produções similares e Brasília com a menor produção dentre as capitais da Região Centro-Oeste do Brasil. Isso poderia ser reflexo de menor investimento nesta especialidade em particular na capital federal do Brasil ou uma menor necessidade de tratamento endodôntico pela população de Brasília. Nenhuma das hipóteses pode ser confirmada por este estudo, mas indicam a necessidade de pesquisas futuras.

Observou-se que os dentes com três ou mais canais radiculares tiveram maior prevalência de tratamento endodôntico do que os dentes com um ou dois canais radiculares. Isso pode ser justificado em razão de os molares (que compõem a maioria dos dentes com três ou mais canais radiculares), em função da complexidade da anatomia de sua coroa, serem mais susceptíveis ao desenvolvimento de lesões cariosas, sendo esta, provavelmente, a principal indicação para o tratamento endodôntico destes dentes ${ }^{17,18}$. Isso pode evidenciar a necessidade de atenção especial dos gestores públicos para ações de prevenção de cárie ou intervenções precoces, quando não há ainda envolvimento pulpar.

Este estudo utilizou DATASUS como fonte de informação devido ao seu fácil acesso e eficiência para obter dados e informações em saúde $^{13,19}$. À medida que o DATASUS trabalha em dados secundários, produzidos para outros fins, é suscetível a erros de digitação e registro, possível subnotificação e inconsistência no preenchimento de dados, como qualquer outra fonte secundária ${ }^{13,19}$. No entanto, como é uma base de dados federal oficial, e os municípios têm a obrigação de preenchê-la, sua importância pode não ser negligenciada ${ }^{20}$. Este sistema de informação pode demonstrar melhor confiabilidade se confrontado com dados epidemiológicos ${ }^{3,21}$, o que também pode ajudar a responder as questões levantadas no estudo.

Os resultados deste estudo sugerem uma preocupação na prestação da assistência odontológica na rede pública dos municípios pesquisados de forma, mais conservadora e especializada, considerando-se o número de tratamentos endodônticos realizados nas diferentes capitais. Esse dado vai de encontro com o senso comum que descreve a assistência odontológica pública brasileira como mutiladora e de baixa qualidade ${ }^{1-3}$.

A Resolução do Conselho Nacional de Educação/Câmara de Educação Superior (CNE/CES) de 2002 institui Diretrizes Curriculares Nacionais (DCN) para os cursos de graduação em Odontologia, objetivando promoção, prevenção, recuperação e reabilitação da saúde, com ênfase nas diretrizes do SUS ${ }^{22,23}$. Entretanto, as instituições de ensino devem estar atentas às mudanças na assistência, fortalecidas com o advento da Política Nacional de Saúde Bucal, para que o egresso esteja em consonância com o perfil epidemiológico da população bem como com as políticas públicas de saúde direcionadas à sua atenção ${ }^{7,8,22,23}$. As estratégias aplicadas na formação profissional devem favorecer uma formação generalista, humanista e comprometida com a sociedade, não apenas contribuindo, mas fortalecendo a mudança de paradigma na assistência odontológica. ${ }^{7,8,22,23}$

\section{CONCLUSÃO}

A produção endodôntica realizada nos Centros de Especialidades Odontológicas nas capitais dos estados da Região Centro-Oeste e do Distrito Federal do Brasil demonstra mudança do paradigma mutilador. A produção foi proporcionalmente maior em Campo Grande e menor em Brasília. O tratamento endodôntico foi 
realizado preferencialmente nos dentes com três ou mais canais radiculares. As instituições de ensino devem estar atentas às mudanças na assistência odontológica prestada no âmbito do SUS para que seus egressos sejam formados em consonância com o perfil epidemiológico da população bem como com as políticas públicas de saúde.

\section{ABSTRACT \\ Provision of endodontics in specialized public centers for dental care in the Center-West of Brazil}

Little is known about the use of public dental services in the center-west region of Brazil, and even less about the number of endodontic treatments. Endodontic procedures through the Brazilian Unified Health System (Sistema Unico de Saúde; SUS) are performed mainly in specialized public centers for dental care, and these data are available from the database of the Brazilian Ministry of Health (DATASUS). This study aimed to determine the number of endodontic procedures performed in state capitals in the center-west of Brazil. We conducted a cross-sectional study with retrospective data collection of all endodontic procedures performed through the SUS from January 2011 to December 2016. Data were obtained from the DATASUS and from the website of the Brazilian Institute of Geography and Statistics and were compared by analysis of variance, using Kolmogorov-Smirnov and Levene's tests followed by Tukey post hoc test at a significance level of $p<0.05$. Campo Grande had the largest number of endodontic treatments per inhabitant (0.063), followed by Cuiabá and Goiânia, with similar rates $(0.028$ and 0.026 respectively), and Brasilia (0.006). Teeth with three or more root canals had significantly more endodontic interventions than teeth with one or two root canals $(p<0.05)$. Higher education institutions must be attentive to the changes in the model of dental care offered by the SUS so that their graduates are trained in accordance with the epidemiological profile of the population as well as with health public policies. Descriptors: Dentistry. Endodontics. Unified Health System.

\section{REFERENCIAS}

1. Paim J, Travassos C, Almeida C, Bahia L, Macinko J. The Brazilian health system: history, advances, and challenges. Lancet. 2011; 377(9779):1778-97.

2. Fernandes JKB, Pinho JR, Queiroz RC, Thomaz EB. Evaluation of oral health indicators in Brazil: a trend towards equity in dental care? Cad Saúde Pública. 2016; 32(2):e00021115.

3. Chaves SCL, Almeida AMFL, Rossi TRA, Santana SF, Barros SG, Santos CML. Oral health policy in Brazil between 2003 and 2014: scenarios, proposals, actions, and outcomes. Ciênc Saúde Colet. 2017; 22(6): 1791-803.

4. Lino PA, Werneck MA, Lucas SD, Abreu $\mathrm{MH}$. Analysis of secondary care in oral health in the state of Minas Gerais, Brazil. Ciênc Saúde Colet. 2014; 19(9):3879-88.

5. Reis CM, Mambrini JV, da Matta-Machado AT, Amaral JH, Werneck MA, Abreu MH. Primary dental care evaluation in Brazil: an item response theory approach. J Public Health Dent. $2017 . \quad$ DOI: 10.1111/jphd.12210.

6. Cunha MA, Lino PA, Santos TR, Vasconcelos M, Lucas SD, Abreu MH. A 15Year Time-series Study of Tooth Extraction in Brazil. Medicine (Baltimore). 2015; 94(47):e1924.

7. Bulgarelli AF, Souza KR, Baumgarten A, Souza JM, Rosing CK, Toassi RFC. Healthcare training with experience in the National Health System: students' perceptions regarding the dentistry course at the Federal University of Rio Grande do Sul (UFRGS), Brazil. Interface Comun Saúde Educ. 2014; 18(49):351-62.

8. Leme PA, Pereira AC, Meneghim Mde C, Mialhe FL. Undergraduate dental students' perspectives about experiences in primary care for their education in the field of health. 
Ciênc Saúde Colet. 2015; 20(4):1255-65.

9. Johnstone M, Parashos P. Endodontics and the ageing patient. Aust Dent J. 2015; 60 Suppl 1:20-7.

10. Castro RD, Rangel ML, da Silva MA, de Lucena BT, Cavalcanti AL, Bonan PR, Oliveira JA. Accessibility to Specialized Public Oral Health Services from the Perspective of Brazilian Users. Int J Environ Res Public Health. 2016; 13(10).

11. Pinheiro RS, Torres TZG. Access to oral health services between Brazilian States. Ciênc Saúde Colet. 2006; 11:999-1010.

12. Pedro FM, Marques A, Pereira TM, Bandeca MC, Lima S, Kuga MC, Tonetto MR, Semenoff-Segundo A, Borges AH. Status of Endodontic Treatment and the Correlations to the Quality of Root Canal Filling and Coronal Restoration. J Contemp Dent Pract. 2016; 17(10):830-6.

13. Celeste RK, Moura FR, Santos CP, Tovo MF. Analysis of outpatient care in Brazilian municipalities with and without specialized dental clinics, 2010. Cad Saúde Pública. 2014; 30(3):511-21.

14. Departamento de Informática do Sistema Único de Saúde (DATASUS). [Acesso em 22 maio 2017]. Disponível em: http://datasus.saude.gov.br/.

15. Traebert J, Suarez CS, Onofri DA, Marcenes W. Prevalence and severity of dental caries and treatment needs in small Brazilian counties. Cad Saúde Pública. 2002; 18(3):817-21.

16. Rocha RACP, Goes PSA. Comparison of access to Oral Health Services between areas covered and not covered by the Family Health Program in Campina Grande, Paraíba State, Brazil. Cad Saúde Pública. 2008; 24(12):2871-80.
17. Gupta A, Duhan J, Wadhwa J. prevalence of three rooted permanent mandibular first molars in Haryana (North Indian) Population. Contemp Clin Dent. 2017; 8(1):38-41.

18. Estrela C, Holland R, Estrela CR, Alencar AH, Sousa-Neto MD, Pécora JD. Characterization of successful root canal treatment. Braz Dent J. 2014; 25(1):3-11.

19. Nóbrega CB, Hoffmann RH, Pereira AC, Meneghim MC. Financing in Brazilian health care system: a recent retrospective and dentistry approach. Ciênc Saúde Colet. 2010; 15 Suppl 1:1763-72.

20. Chaves SC, Cruz DN, Barros SG, Figueiredo AL. Assessing the supply and use of secondary care in specialized dental clinics in Bahia State, Brazil. Cad Saúde Pública. 2011; 27(1):143-54.

21. Thomaz EB, Sousa GM, Queiroz RC, Coimbra LC. Evaluating productivity targets achievement in Dental Specialty Centers from Maranhão State, Brazil, 2011. Epidemiol Serv Saúde. 2016; 25(4):807-18.

22. Forte FDS, Pessoa TRRF, Freitas CHSM, Pereira CAL, Carvalho Junior PM. Reorienting dental education: the preceptor's view of supervised internship in the Brazilian Health System (SUS). Interface Comun Saúde Educ. 2015; 19 Supl 1:831-43.

23. Emmi DT, Silva DMC, Barroso RFF. Integrated teaching to service experience in Health professional education: perception of dentistry students and graduates. Interface Comun Saúde Educ.. 2018; 22(64):223-36.

\section{Correspondência para:}

Thiago Machado Pereira e-mail: thiagomachado@hotmail.co.uk Av. Manoel José de Arruda, 3100 Jardim Europa 78065-900 Cuiabá/MT 